\title{
Laboreal
}

Volume $14 \mathrm{~N}^{\circ} 1$ | 2018

$\mathrm{O}$ regresso ao emprego após um acidente de trabalho

\section{Volver sobre Gilbert Simondon y su libro inicial}

Um retorno a Gilbert Simondon e à sua obra inicial

Un retour à Gilbert Simondon et à son cuvre initiale

A return to Gilbert Simondon and to his initial work

Jacques Leplat

Traductor. Patricio Nusshold

\section{OpenEdition}

\section{Journals}

Edición electrónica

URL: http://journals.openedition.org/laboreal/545

DOI: 10.4000/laboreal.545

ISSN: 1646-5237

Editor

Universidade do Porto

Referencia electrónica

Jacques Leplat, «Volver sobre Gilbert Simondon y su libro inicial », Laboreal [En línea], Volume $14 \mathrm{~N}^{0} 1$ ।

2018, Publicado el 01 julio 2018, consultado el 24 septiembre 2020. URL : http://

journals.openedition.org/laboreal/545; DOI : https://doi.org/10.4000/laboreal.545

Este documento fue generado automáticamente el 24 septiembre 2020

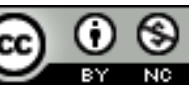

Laboreal está licenciado com uma Licença Creative Commons - Atribuição-NãoComercial 4.0 Internacional. 


\title{
Volver sobre Gilbert Simondon y su libro inicial
}

\author{
Um retorno a Gilbert Simondon e à sua obra inicial \\ Un retour à Gilbert Simondon et à son œuvre initiale \\ A return to Gilbert Simondon and to his initial work
}

Jacques Leplat

Tradución : Patricio Nusshold

\section{REFERENCIA}

Comentario del texto de Simondon, G. (1969). Introduction. In G. Simondon. Du mode d'existence des objets techniques (pp. 9-16). Paris: Aubier.

\section{NOTA DEL EDITOR}

http://dx.doi.org/10.15667/laborealxiv0118jles

Manuscrito recibido en: Enero /2017

Aceptado tras peritaje: Febrero /2017

Agradezco muy fuertemente a Marianne Lacomblez y Régis Ouvrier Bonnaz por la relectura que han realizado de mi texto inicial, para ponerlo a la altura de las normas de la revista y facilitar la lectura a los futuros lectores.

1 Nadie que se interese a la historia del trabajo se sorprenderá de ver el nombre de Simondon en un texto de la revista Laboreal, generalmente orientada a cuestiones relativas a las áreas de la psicología del trabajo y la ergonomía. Como este autor escribe en la introducción de su libro «El modo de existencia de los objetos técnicos» [1], «el hombre es el organizador permanente de una sociedad de objetos técnicos que tienen necesidad de él como los músicos tienen necesidad del director de orquesta.» (p.33). La 
historia de la ergonomía de la actividad, como la de la psicología del trabajo, aporta numerosas ilustraciones de esta declaración, desde los primeros tiempos de la afirmación del abordaje que les es común (Ombredane et Faverge, 1955 ; Leplat et Cuny, 1977 ; de Monmollin, 1984; Wisner, 1985; Rabardel, 1995). El nombre de Gilbert Simondon se encuentra asociado al de los objetos técnicos. Sin embargo, no hay que reducir la obra de Simondon a este único aspecto. Es posible constatar, desde la lectura de las primeras páginas de este libro, que sus análisis se desarrollan en un marco más amplio.

\section{Gilbert Simondon, un autor mayor, original, pero apreciado tardíamente}

2 Antes de realizar este comentario, recordaré que Simondon comenzó su carrera en la Universidad de Poitiers, que abandonó luego por la de la Sorbona. Como se puede constatar en su biografía retomada en este libro, Simondon es el autor de un gran número de publicaciones sobre asuntos diversos, aunque su interés por los temas ligados a las relaciones entre el hombre y el trabajo fueron preponderantes, habiéndose manifestado de varios modos: a través colaboraciones con docentes, conferencias, participaciones en jurados de tesis y de numerosos congresos.

3 Tuve la suerte de conocerlo al inicio de su carrera. Luego, de encontrarlo a menudo en su Laboratorio en el Instituto de Psicología en la calle Serpente (Sorbona). Nunca olvidaré las visitas guiadas que me hizo hacer en su sala de experimentación en la cual se encontraban una cierta cantidad de objetos que figuran en la «mesa de trabajo» al inicio del libro que analizamos aquí. Cada uno de esos objetos extremadamente variados tenía una historia que a él le gustaba contar, disponible para responder a todas las preguntas.

Gilbert Simondon (1929-1989) es entonces un autor mayor y original en el campo de las investigaciones sobre las relaciones entre la cultura y la técnica. El presente libro ocupa un lugar central, reconocido por la riqueza del análisis propuesto, y por las numerosas pistas de investigación que abre. El interés que ha recibido es, por otro lado, renovado hoy en día en el marco de numerosos proyectos. Algunos hacen así referencia a un «redescubrimiento de la obra de Simondon». Es verdad si su originalidad y valor fueron rápidamente reconocidos por sus pares, la obra no conquistó un público amplio, seguramente desalentado por los pasajes por momentos muy técnicos del libro. De hecho, hicieron falta años para que esta contribución encuentre finalmente la audiencia que merece, probablemente favorecida por el interés de algunos grandes lectores que constituyen «el fondo a partir del quel Simondon ha construído, a través del diálogo directo con la epistemología de Canguilhem, la fenomenología de Maurice Merleau-Ponty y la cibernética de Norbert Wiener, la originalidad de un pensamiento que autoriza una perspectiva que se podría llamar ecológica, y cuya fuerza debe ser necesariamente considerada hoy en día. » (Bardin, 2014, p. 26).

5 A modo de ejemplo de los esfuerzos realizados para valorizar la obra de G. Simondon, hace ya un cuarto de siglo, en 1992, Hubert Curien, antiguo director del Centro Nacional de la Investigación Científica (CNRS) y del Centro Nacional de Estudios Espaciales (CNES), durante un coloquio organizado por el Collège internacional de filosofía expresaba: «Estoy particularmente feliz de introducir un encuentro que se esfuerza en 
analizar y rememorar el pensamiento de Gilbert Simondon. Este pensamiento, tan necesario, tan fecundo para nuestro tiempo, tan ejemplarmente actual, debe ser retirado de un injusto olvido. (...) G.S. era un hombre de una gran cultura científica, particularmente en física y biología (...). Se trataba también de un investigador ejemplar de la transformación cultural, de las realidades psicológicas y sociales: no recortaba artificialmente el mundo a partir de la tan lamentable separación entre ciencias de la naturaleza, por un lado, y ciencias del espíritu, por el otro.»

\section{Génesis del objeto técnico: el proceso de concretización}

6 El análisis exhaustivo del libro excedería la finalidad habitual de esta rúbrica de la revista. Privilegiaremos entonces aquí, en relación a la introducción, aquello que retuvimos del primer capítulo y de la conclusión del libro.

El primer capítulo vuelve en realidad a la introducción retomando los temas a menudo desarrollados en las investigaciones relativas a las situaciones de trabajo. Aquí el autor elige el motor del automóvil como ejemplo de objeto técnico. Subraya :

8 En el motor antiguo, cada elemento interviene en un determinado momento del ciclo, y por lo tanto se supone que no actúa sobre los otros elementos», «en un motor actual cada pieza importante está tan ligada a las otras por medio de intercambios recíprocos de energía que no puede ser otra que lo que es.» «Se podría decir que el motor actual es un motor concreto, mientras que el motor antiguo es un motor abstracto.» La esencia de la concretización del objeto técnico es la organización de sub-conjuntos funcionales en el funcionamiento total (p.43, traducción libre).

9 En esta lógica, Simondon estudia entonces, sucesivamente: la evolución técnica, el ritmo del progreso técnico y las tradiciones técnicas. Insiste mucho sobre las modalidades y las consecuencias de la concretización de los objetos técnicos, así como su explotación. Según él, el oficio recurre al estado primitivo de la evolución de los objetos técnicos, o sea al estado abstracto, y la industria saca provecho de su estadio concreto. Las modalidades de este pasaje se examinan en detalle y se discuten a partir de ejemplos precisos que permiten identificar estilos de evolución. E indica entonces que la construcción de un objeto técnico determinado puede volverse industrial cuando ese objeto se vuelve concreto, a saber: cuando el conocimiento subyacente al aprendizaje constructivo y el de la mirada científica son análogas. También dice:

10 (...) existen entonces dos tipos de perfeccionamientos (...) los perfeccionamientos menores estorban a los mayores, porque pueden enmascarar las verdaderas imperfecciones de un objeto técnico, compensando a través de artificios inesenciales, integrados de modo incompleto al funcionamiento de conjunto, los verdaderos antagonismos ... (...) Las verdaderas etapas del perfeccionamiento del objeto técnico se hacen por medio de mutaciones, pero por mutaciones orientadas» (p. 59, 60 y 61).

11 Y agrega, más adelante, al momento de brindar otro ejemplo, el del diodo:

12 «La concretización da al objeto técnico un lugar intermedio entre el objeto natural y la representación científica. El objeto técnico abstracto, es decir primitivo, está muy lejos de constituir un sistema natural. Es la traducción en la materia de un conjunto de nociones y principios científicos (...) Por el contrario, el objeto técnico concreto, es decir evolucionado, se aproxima al modo de existencia de los objetos naturales, tiende a 
la coherencia interna, a la cerrazón del sistema de causas y efectos que se ejercen circularmente en el interior de su recinto (...). Este objeto, al evolucionar, pierde su carácter de artificialidad: la artificialidad esencial de un objeto reside en el hecho de que el hombre debe intervenir para mantener este objeto en la existencia protegiéndolo contra el mundo natural, dándole un estatuto aparte de existencia (p. 67, traducción libre).

\section{Objeto técnico y trabajo}

La importancia de la contribución de Simondon en toda reflexión sobre las relaciones entre objeto técnico y trabajo humano es comprensible. En el primer párrafo de la conclusión del libro, escribe, por cierto:

Hasta hoy la realidad del objeto técnico pasó a un segundo plano, detrás de la del trabajo humano. El objeto técnico ha sido aprehendido a través del trabajo humano, pensado y juzgado como instrumento, adyuvante o producto del trabajo. Ahora bien, habría que poder operar, a favor del mismo hombre, una inversión que permitiera a lo que hay de humano en el objeto técnico aparecer directamente, sin pasar a través de la relación de trabajo. Es el trabajo lo que debe ser conocido como fase de la tecnicidad, y no la tecnicidad como fase de trabajo, porque la tecnicidad es el conjunto del cual el trabajo es una parte, y no a la inversa (p. 257, traducción libre).

Estos temas serán retomados y profundizadas más tarde. Se pueden ver más desarrollados en un libro que reúne textos de Simondon (2014). Sin embargo, aquí ya nos dice:

(...) el trabajo puede ser comprendido como aspecto de la operación técnica, que no se reduce al trabajo. Sólo hay trabajo cuando el hombre debe donar su organismo como portador de herramientas, es decir, cuando el hombre debe acompañar a través de la actividad de su organismo, de su unidad somato-psíquica, el despliegue etapa por etapa de la relación hombre-naturaleza. El trabajo es la actividad por la cual el hombre realiza en sí mismo la mediación entre la especie humana y la naturaleza; decimos que en ese caso el hombre opera como portador de herramientas porque en esa actividad actúa sobre la naturaleza y sigue, paso a paso, gesto por gesto esta acción. Hay trabajo cuando el hombre no puede confiar al objeto técnico la función de mediación entre la especie y la naturaleza, y debe realizar él mismo, a través de su cuerpo, su pensamiento, su acción, esta función de relación. El hombre presta entonces su propia indivdualidad de ser vivo para organizar esta operación ; aquí es donde es portador de herramientas. Por el contrario, cuando el objeto técnico está concretizado, la mezcla de naturaleza y de hombre está constituida en el nivel de ese objeto; la operación sobre el ser técnico no es exactamente un trabajo. En efecto, en el trabajo, el hombre coincide con una realidad que no es humana, se pliega a esta realidad, se desliza de alguna manera entre la realidad natural y la intención humana; el hombre, en el trabajo, modela la materia según una forma; llega con esta forma, que es una intención del resultado, una predeterminación de lo que hay que obtener al término de la obra según las necesidades preexistentes. Esta forma-intención no forma parte de la materia sobre la que trata el trabajo; expresa una utilidad o una necesidad para el hombre, pero no se desprende de la naturaleza. La actividad de trabajo es lo que establece el vínculo entre la materia natural y la forma, de proveniencia humana; el trabajo es una actividad que 
llega a hacer coincidir, a hacer sinérgicas, dos realidades tan heterogéneas como la materia y la forma (p. 257 y 258, traducción libre).

\section{Del modo de existencia de los objetos técnicos a los resultados de su funcionamiento}

Por último, lamentaríamos no evocar, en esta breve presentación, la perspectiva de investigación que Simondon traza al indicar en qué medida el estudio del modo de existencia de los objetos técnicos debería prolongarse en el estudio de los resultados de su funcionamiento, y de las actitudes del hombre ante esos objetos técnicos:

La actividad técnica, al edificar el mundo de los objetos técnicos y al generalizar la mediación objetiva entre hombre y naturaleza, aproxima el hombre a la naturaleza según un vínculo mucho más rico y mejor definido que el de la reacción específica del trabajo colectivo. A través del esquematismo técnico se instituye una convertibilidad de lo humano en natural y de lo natural en humano (...). La percepción corresponde al cuestionamiento directo del hombre viviente por parte del mundo natural. La ciencia corresponde al mismo cuestionamiento a través del universo técnico. La sensación basta para el trabajo sin obstáculo; la percepción corresponde al problema que surge en el nivel del trabajo. Por el contrario, mientras que las técnicas sean exitosas, el pensamiento científico no está invitado a nacer. Cuando las técnicas fallan, la ciencia está cerca. La ciencia corresponde a una problemática formulada en el nivel de las técnicas, y que no puede encontrar la solución en el nivel técnico. La técnica interviene entre percepción y ciencia para hacer cambiar de nivel; provee los esquemas, las representaciones, los medios de control, las mediaciones entre hombre y naturaleza. El objeto técnico convertido en separable puede ser agrupado con otros objetos técnicos según tal o cual montaje: el mundo técnico ofrece una disponibilidad indefinida de agrupamientos y conexiones (...). Construir un objeto técnico es preparar una disponibilidad (p. 261 y 262, traducción libre).

19 Concluyamos junto a Gilbert Simondon: «El objeto que sale de la invención técnica lleva consigo algo del ser que lo ha producido, expresa aquello de ese ser que está menos ligado a un hic et nunc» (p. 263).

Es verdad efectivamente que nunca se termina realmente de leer un texto de Simondon y en particular este primer texto que escribió, más que los otros. La lectura es por momentos difícil y requiere perseverancia. Podemos estar de acuerdo en que todo está preparado para un nuevo comienzo.

21 Título original en francés « Du mode d'existence des objets techniques ». París: Editions Flammarion. Primera edición en francés en 1958; segunda edición en 1969. Edición en castellano en 2007, Buenos Aires: Prometeo Libros, utilizada para el presente texto en castellano. 


\section{BIBLIOGRAFÍA}

Bardin, A. (2014). Le monde diplomatique, Décembre.

Curien, H. et al. (1992). Une pensée de l'individualisation et de la technique. Paris : Albin Michel.

Leplat, J. et Cuny, X. (1977). Introduction à la psychologie du travail. Paris : P.U.F.

Montmollin (de), M. (1984). L'intelligence de la tâche. Peter Lang : Bern.

Ombredane, A. et Faverge, J.-M. (1955). $L^{1}$ analyse du travail. Paris : P.U.F.

Rabardel P. (1995). Les hommes et les technologies. Paris : Armand Colin.

Simondon, G. (1969). Du mode d'existence des objets techniques. Paris : Aubier.

Simondon, G. (2014). Sur la technique (1953-1983) Paris : P.U.F.

\section{NOTAS}

1. Título original en francés «Du mode d'existence des objets techniques». París: Editions Flammarion. Primera edición en francés en 1958; segunda edición en 1969. Edición en castellano en 2007, Buenos Aires: Prometeo Libros, utilizada para el presente texto en castellano.

\section{AUTORES}

\section{JACQUES LEPLAT}

École Pratique des Hautes Études

Grupo de pesquisa e de estudo da história do trabalho e da orientação (GRESHTO)

Centro de pesquisa do trabalho e do desenvolvimento (CRTD)

Conservatório Nacional das Artes e Ofícios (CNAM)

41, Rue Gay-Lussac 75005 Paris, France

Jacques.leplat@wanadoo.fr 